04

\title{
Структура призондового слоя в газоразрядной плазме при произвольной ориентации плоского зонда относительно электрического поля в плазме
}

\author{
(C) О. Мурильо, ${ }^{1}$ А.С. Мустафаев, ${ }^{1}$ В.С. Сухомлинов ${ }^{2}$ \\ ${ }^{1}$ Санкт-Петербургский горный университет, \\ 199106 Санкт-Петербург, Россия \\ ${ }^{2}$ Санкт-Петербургский государственный университет, фризический фракультет, \\ 198504 Санкт-Петербург, Россия \\ e-mail: rectorat@spmi.ru, v_sukhomlinov@mail.ru
}

Поступило в Редакцию 15 февраля 2019 г.

В окончательной редакции 19 апреля 2019 г.

Принято к публикации 29 апреля 2019 г.

\begin{abstract}
Исследована структура пристеночного слоя газового разряда около плоской поверхности, находящейся при отрицательном потенциале, большем средней энергии электронов. Показано, что при условиях, когда средняя энергия ионов в плазме много меньше таковой для электронов, параметры пристеночного слоя слабо зависят от взаимной ориентации нормали к поверхности и электрического поля в плазме для произвольного отношения радиуса Дебая к длине свободного пробега ионов относительно резонансной перезарядки. Обнаружено, что для инертных газов Не, Ar при отношении электрического поля к давлению $\left(\frac{R}{P}\right)$ более $10 \frac{\mathrm{V}}{\mathrm{cm} \cdot \mathrm{Torr}}$ в плазме неучет процесса ионизации в возмущенном пристеночном слое может приводить к существенным ошибкам в расчете его параметров. Показано, что наличие ионизации приводит к росту электрического поля в пристеночном слое и, как следствие, увеличению средней скорости ионов на границе квазинейтрального „предслоя“ и части пристеночного слоя, где существенно нарушается квазинейтральность. Параметры пристеночного слоя, где существенно нарушается квазинейтральность, зависят от частоты ионизации существенно меньше, чем соответствующие параметры квазинейтрального „предслоя“. Получено соотношение для определения концентрации заряженных частиц в невозмущенной плазме по ионному току насыщения с учетом реальной функции распределения ионов по энергиям в плазме, ионизации в „предслоя“ и части пристеночного слоя, где существенно нарушается квазинейтральность.
\end{abstract}

Ключевые слова: критерий Бома, пристеночный слой, уравнение Больцмана, газоразрядная плазма.

DOI: $10.21883 / J T F .2019 .10 .48170 .54-19$

\section{Введение и постановка задачи}

Пристеночные слои при отрицательном потенциале стенки образуются, в частности, около границ объема плазмы, вблизи электрических зондов, около катода. Поскольку структура слоя определяет функцию распределения ионов (ФРИ), бомбардирующих стенку, ее определение имеет большое значение для различных приложений, среди которых вопросы исследования плазмохимических реакций, идущих с участием ионов; определение подвижности ионов в плазменном объекте; процессы нагрева нейтральной компоненты плазмы; современные плазменные нанотехнологии: тонкая очистка ионами поверхности изделий, технология создания рельефов на поверхности за счет избирательного травления при бомбардировке потоками ионов [1-4] и ряд других. Знание структуры пристеночного слоя необходимо и в теории зондов при определении концентрации заряженных частиц в плазме по так называемому току насыщения [5,6]. Отметим, что кинетическая теория для вычисления этого тока, использующая известный критерий Бома [7-10], также основывается на знании ФРИ в возмущен- ном слое и до сих пор окончательно не разработана [8].

Принято считать, что возмущенный пристеночный слой (ВПС) в рассматриваемой ситуации имеет следующую структуру. Между невозмущенной плазмой и пристеночным слоем (ПС), в котором существенно нарушение квазинейтральности, расположен так называемый квазинейтральный „предслой“, в котором и происходит ускорение ионов до скорости, обеспечивающей монотонность потенциала в ПС [8-12]. Условие, которое определяет эту скорость называется критерием Бома [7,13-22] (хотя сам Бом в своей работе рассматривал только бесстолкновительный $\mathrm{B \Pi C).}$

Необходимость в критерии Бома обусловлена отсутствием теории, описывающей параметры потока ионов в квазинейтральном „предслое“. Более или менее адекватные теории, учитывающие, в частности, перезарядку и ионизацию в ПС, построены для собственно ПС, однако для самосогласованного решения данной задачи в замкнутом виде требуются граничные условия, которые и ставятся, в частности, на границе ПС и „предслоя“. В отсутствие информации о ФРИ на этой 
границе, для преодоления этой трудности ряд авторов предлагают различные способы сшивки решения для ПС и „Предслоя“ [23-27], а также делают различные нереалистичные предположения о поведении поля вблизи границы ПС и „Предслоя“, например, равенство нулю производной и (или) самого поля [22,24]. Как будет видно из дальнейшего, подобные допущения являются слишком грубыми и приводят к существенному искажению структуры ВПС, особенно „Предслоя“.

В работах [28,29] была решена задача о структуре ВПС при условии отсутствия ионизации в ВПС и для ситуации, когда электрическое поле в плазме антипараллельно внешней нормали к плоской возмущающей поверхности. Вместе с тем при зондовых измерениях анизотропных ФР заряженных частиц методом плоского одностороннего зонда необходимо проводить измерения при произвольной ориентации зонда относительно электрического поля в плазме [30-32]. Кроме того, в неравновесной газоразрядной плазме зачастую реализуются условия, требующие учета ионизации в ВПС, что, как будет показано в настоящей работе, существенно влияет на его структуру. Развитию теории ВПС с учетом этих особенностей и посвящена настоящая работа.

\section{Построение теории ВПС}

Рассмотрим задачу о формировании электрического поля и пространственных концентраций заряженных частиц в ВПС при отрицательном потенциале плоского зонда с позиций кинетического подхода. Предположим, что в газоразрядной плазме есть плоская поверхность, находящаяся под некоторым отрицательным относительно плазмы потенциалом $U$. Тогда вблизи этой поверхности образуется ВПС. Пусть на некотором расстоянии от стенки параметры плазмы мало отличаются от таковых для случая $U=0$. Будем считать эту плоскость границей невозмущенной плазмы и ВПС. Введем систему прямоугольных декартовых координат, таким образом, что плоскость этой $X Y$ системы совпадает с введенной выше плоской границей. Будем считать, что ионы в невозмущенной плазме и в ВПС испытывают только резонансную перезарядку при столкновениях с нейтралами. Качественно структура ВПС, согласно вышесказанному, имеет вид, представленный на рис. 1. Мы не будем искусственно разбивать возмущенный слой около стенки на квазинейтральный „предслой“ и собственно ПС, где квазинейтральность существенно нарушается. Существование этих частей ВПС возникнет естественно в процессе решения поставленной задачи.

Для ее решения необходимо сформулировать самосогласованную систему уравнений - интегро-дифференциального уравнения Больцмана и уравнения Пуассона. Будем сначала считать, что сечение $\sigma$ перезарядки иона на собственном атоме не зависит от энергии иона, а ионы - однозарядные. Очевидно, что в рассматриваемой ситуации параметры плазмы зависят только от координаты $z$. Поскольку ФРИ во введенной системе

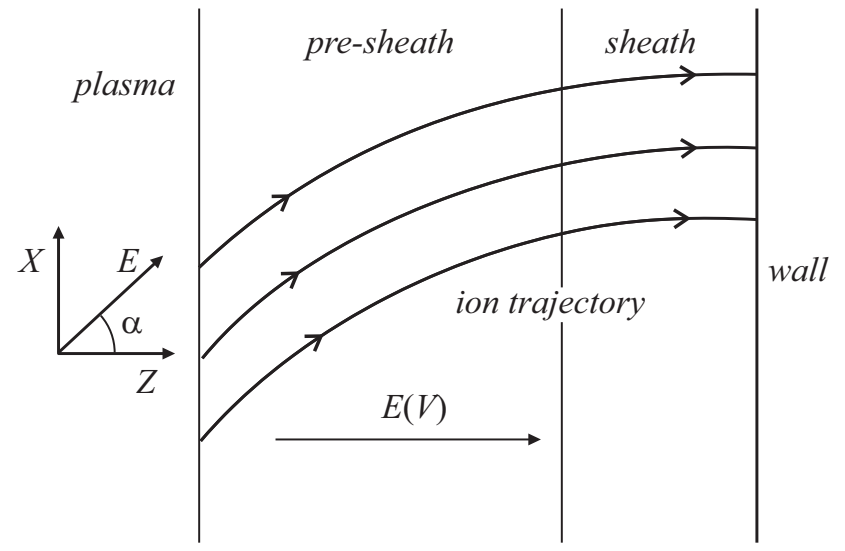

Рис. 1. Структура ВПС при произвольном направлении электрического поля в невозмущенной плазме.

координат не зависит от $x, y$ и $v_{y}$ (проекции скорости иона на ось $y$ ), то, сохраняя принятые ранее обозначения, запишем уравнение Больцмана для ФРИ $f_{i}$ в виде

$$
\begin{aligned}
& v_{z} \frac{\partial f_{i}}{\partial z}+\frac{e E(z)}{M} \frac{\partial f_{i}}{\partial z}+\frac{e E \cdot \sin \alpha}{M} \frac{\partial f_{i}}{\partial z} \\
& =\delta\left(\frac{v^{2}}{2}\right) v\left[N_{a} \sigma I_{0}(z)+v_{i} n_{e}(z)\right]-N_{a} \sigma v f_{i},
\end{aligned}
$$

где $I_{0}(z)=\int_{0}^{\infty} v^{\prime} f_{i}\left(v^{\prime}, z\right) d v^{\prime}-$ плотность потока ионов; $\alpha$ - угол между электрическим полем в невозмущенной плазме и внешней нормалью к поверхности (рис. 1); $v_{z}, v_{x}-Z, X$-компоненты скорости иона во введенной системе координат соответственно; $N_{a}, n_{e}(z)-$ концентрации нейтральных частиц и электронов соответственно; $M, e$ - масса иона и заряд электрона соответственно; $v_{i}$ - частота ионизации, которую мы считаем не зависящей от координаты $z ; E(z)-Z$-компонента электрического поля в ВПС, так, что

$$
E(0)=E \cdot \cos \alpha .
$$

Перейдем в уравнении (1) к независимым переменным $z, v, \mu=\cos \theta$. Тогда это уравнение перепишется в виде

$$
\begin{aligned}
& v \mu \frac{\partial f_{i}}{\partial z}+\frac{e}{M}\left[E \cdot \sin \alpha\left(\sqrt{1-\mu^{2}} \frac{\partial f_{i}}{\partial v}-\frac{\mu \sqrt{1-\mu^{2}}}{v} \frac{\partial f_{i}}{\partial \mu}\right)\right. \\
& \left.+E(z)\left(\mu \frac{\partial f_{i}}{\partial v}+\frac{1-\mu^{2}}{v} \frac{\partial f_{i}}{\partial \mu}\right)\right] \\
& =\delta\left(\frac{v^{2}}{2}\right) v\left[N_{a} \sigma I_{0}(z)+v_{i} n_{e}(z)\right]-N_{a} \sigma v f_{i}
\end{aligned}
$$

Вполне очевидно, что если ионы испытывают только резонансную перезарядку на атомах, то при произвольном поле $E(z)$ все они двигаются по параллельным траекториям (рис. 1). Таким образом, выполняется

$$
f_{i}(z, v, \mu)=\varphi(z, v) \delta[\mu-\mu(z)],
$$


где $\varphi(z, v), \mu(z)$ - некоторые пока неизвестные функции, а $\mu(0)=\cos \alpha$. Подставим (4) в (3) и проинтегрируем результат по $\mu$ от -1 до 1 . Затем умножим (3) на $\mu$ и также проинтегрируем. Используя два полученных соотношения, легко получить, что

$$
E \cdot \sin \alpha \sqrt{1-\mu(z)^{2}}+E(z) \mu(z)=\sqrt{(E \cdot \sin \alpha)^{2}+E(z)^{2}} .
$$

Из (3) и (5) получаем уравнение

$$
\begin{aligned}
& v \frac{E(z)}{\sqrt{(E \cdot \sin \alpha)^{2}+E(z)^{2}}} \frac{\partial f_{i}}{\partial z} \\
& +\frac{e}{M} \sqrt{(E \cdot \sin \alpha)^{2}+E(z)^{2}} \frac{\partial f_{i}}{\partial v} \\
& =\delta\left(\frac{v^{2}}{2}\right) v\left[N_{a} \sigma I_{0}(z)+v_{i} n_{e}(z)\right]-N_{a} \sigma v f_{i} .
\end{aligned}
$$

Поскольку ФРИ не зависит от координаты $x$, то уравнение (6), записанное в переменных $v, L$ (где $L-$ расстояние, отсчитываемое от границы плазмы вдоль траектории иона), тождественно уравнению

$$
\begin{aligned}
& v \frac{\partial f_{i}}{\partial L}+\frac{e}{M} \sqrt{(E \cdot \sin \alpha)^{2}+E(L)^{2}} \frac{\partial f_{i}}{\partial v} \\
& =\delta\left(\frac{v^{2}}{2}\right) v\left[N_{a} \sigma I_{0}(L)+v_{i} n_{e}(L)\right]-N_{a} \sigma v f_{i} .
\end{aligned}
$$

При этом

$$
\frac{d L}{d z}=\sqrt{1+\frac{(E \cdot \sin \alpha)^{2}}{E(L)^{2}}} .
$$

Уравнение (7) заменой переменных приводится к неоднородному дифференциальному уравнению первого порядка. Действуя аналогично тому, как это было сделано в $[28,29]$, можно получить

$$
\begin{aligned}
& f_{i}(\varepsilon, l)=\exp (-l) f_{i 0}\left(\varepsilon-\frac{\omega^{2}(0, l)}{2}\right) \\
& +\exp [-(L-y(\varepsilon, l))] \frac{M}{e \lambda_{i} E(y(\varepsilon, l))} \\
& \times\left[I_{0}(y(\varepsilon, l))+\lambda_{i} v_{i} n_{e}(y(\varepsilon, l))\right],
\end{aligned}
$$

где $\quad l=\frac{L}{\lambda_{i}} ; \quad \varepsilon=\frac{v^{2}}{2 v_{i}^{2}} ; \quad v_{i}=\frac{e E \lambda_{i}}{M} ; \quad f_{i 0}(\varepsilon)=f_{i}(\varepsilon, 0)$; $\kappa_{2}=\frac{k T_{e}}{e E \lambda_{i}} ; \quad \omega\left(l_{1}, l_{2}\right)=\sqrt{2 \kappa_{2}\left[\bar{V}\left(l_{2}\right)-\bar{V}\left(l_{1}\right)\right]} ; \quad \bar{V}(V)=$ $=\int_{0}^{V} \frac{1}{\sqrt{1-a\left(V^{\prime}\right)^{2} \sin ^{2} \alpha}} d V^{\prime} ; V=-\frac{e U}{k T_{e}} \geq 0 ; y(\varepsilon, l)-$ решение уравнения $x \sqrt{2 \varepsilon}=\omega(y, l) ; a(V)=\frac{E}{\sqrt{(E \cdot \sin \alpha)^{2}+E(V)^{2}}}$; $\lambda_{i}$ - длина пробега иона относительно перезарядки.

Для нахождения $I_{0}(l)$ умножим соотношение (9) на $v$ и проинтегрируем по $d v$. В результате получим интегральное уравнение для величины $\Phi_{0}(l)=I_{0}(l) e^{l}$

$$
\Phi_{0}(l)=I_{0}+\int_{0}^{l}\left[\Phi_{0}(x)+\lambda_{i} v_{i} n_{e}(x) e^{x}\right] d x,
$$

где $I_{0}=I_{0}(0)$.

Решая уравнение (10), находим

$$
I_{0}(l)=I_{0}+\lambda_{i} v_{i} \int_{0}^{l} n_{e}(x) d x
$$

Для вычисления концентрации ионов $n_{i}(l)$ подставим (11) в (9) и проинтегрируем по $d v$. Имеем

$$
\begin{aligned}
& n_{i}(l)=n_{i 1}(l)+n_{i 2}(l) ; n_{i 1}(l)=e^{-l} \int_{0}^{\infty} \frac{f_{i 0}(\varepsilon)}{\sqrt{2 \varepsilon+\omega^{2}(0, l)}} d \varepsilon ; \\
& n_{i 2}(l)=\frac{1}{v_{i}} \int_{0}^{l} \frac{\exp [-[l-x]]}{\omega(x, l)} \\
& \quad \times\left\{\left[I_{0}+\lambda_{i} v_{i} \int_{0}^{x} n_{e}\left(x^{\prime}\right) d x^{\prime}\right]+\lambda_{i} v_{i} n_{e}(x)\right\} d x .
\end{aligned}
$$

Введем параметр $\kappa_{3}$ :

$$
\kappa_{3}=\frac{\lambda_{i} v_{i} n_{0}}{I_{0}}=\frac{\lambda_{i} v_{i}}{v_{i d}}
$$

и перейдем от переменной $x$ к $V$. Тогда, используя в качестве $f_{i 0}(\varepsilon)$ ФРИ полученную в работе [30]:

$$
\begin{gathered}
f_{i 0}(v)=C \exp \left(-\frac{v^{2}}{2 v_{i}^{2}}\right) \operatorname{erfc}\left(-\sqrt{\left(\varepsilon_{0}-1\right) \frac{v^{2}}{2 v_{i}^{2}}}\right) ; \\
v_{i}=\sqrt{\frac{e E \lambda_{i}}{M}} ; \quad v \geq 0 \\
C=\frac{I_{0}}{v_{i}^{2}\left(1+\sqrt{\frac{\varepsilon_{0}-1}{\varepsilon_{0}}}\right)} \approx \frac{I_{0}}{2 v_{i}^{2}}
\end{gathered}
$$

относительную концентрацию ионов $N_{i}(\bar{V})=\frac{n_{e}(\bar{V})}{n_{0}}$, можно записать в виде

$$
\begin{aligned}
& N_{i}(\bar{V})=\bar{N}_{i}[y(\bar{V})]=\bar{N}_{1 i v}(\bar{V})+\bar{N}_{2 i v}(\bar{V}) ; \\
& \bar{N}_{1 i v}(\bar{V})=\exp \left[-R(\bar{V})+\kappa_{2} \bar{V}\right] \operatorname{erfc}\left(\sqrt{\kappa_{2} \bar{V}}\right) ; \\
& \bar{N}_{2 i v}(\bar{V})=\sqrt{\frac{\kappa_{2}}{\pi}} \exp [-R(\bar{V})] \int_{0}^{\bar{V}} \exp \left[R\left(V^{\prime}\right)\right] \frac{a\left(V^{\prime}\right)}{\sqrt{\bar{V}-V^{\prime}}} \\
& \times\left\{1+\kappa_{3}\left[\kappa_{2} \int_{0}^{V^{\prime}} \widetilde{N}_{e}\left(V^{\prime \prime}\right) a\left(V^{\prime \prime}\right) d V^{\prime \prime}+N_{e}\left(V^{\prime}\right)\right]\right\} d V^{\prime} \\
& \bar{N}_{i}(0)=1,
\end{aligned}
$$


Вводя в уравнение Пуассона вместо координаты $x$ переменную $\bar{V}$ и, действуя аналогично тому, как это сделано в $[28,29]$, можно записать

$$
\begin{aligned}
\frac{d\left[\frac{\sqrt{1-a(\bar{V})^{2} \sin \alpha^{2}}}{a(\bar{a})}\right]^{2}}{d \bar{V}}= & \frac{2 \kappa_{2}^{2}}{\kappa_{1}^{2}}\left\{N_{i}(\bar{V})-N_{e}(\bar{V})\right\} \\
& \times \sqrt{1-a(\bar{V})^{2} \sin ^{2} \alpha},
\end{aligned}
$$

где $N_{e}(V)$ - относительная концентрация электронов и $N_{e}(0)=1$. Если перейти в уравнении $(16)$ к независимой переменной $V$, то нелинейное интегро-дифференциальное уравнение приводится к системе трех дифференциальных уравнений и одного интегро-дифференциального (более простого, чем (16)):

$$
\begin{gathered}
R^{\prime}(V)=\frac{\kappa_{2} \sqrt{F(V)+\sin ^{2} \alpha}}{F(V)} ; \\
E^{\prime}(V)=\frac{2 \kappa_{2}^{2}}{\kappa_{1}^{2}}\left\{N_{i}(V)-N_{e}(V)\right\} ; \\
G^{\prime}(V)=\frac{N_{e}(V)}{\sqrt{F(V)+\sin ^{2} \alpha}} ; \\
\bar{N}_{2 i v}(V)=\sqrt{\frac{\kappa_{2}}{\pi}} \exp [-R(V)] \int_{0}^{V} \exp \left[R\left(V^{\prime}\right)\right] \\
\times \frac{1}{\sqrt{\left[\bar{V}\left(V^{\prime}\right)-V^{\prime}\right]\left[\bar{F}\left(V^{\prime}\right)+\sin ^{2} \alpha\right]}} \\
\times\left\{1+\kappa_{3}\left[\kappa_{2} \bar{G}\left(V^{\prime}\right)+\tilde{v}_{i}\left(V^{\prime}\right) N_{e}\left(V^{\prime}\right)\right]\right\} d V^{\prime} ;
\end{gathered}
$$

где

$$
\bar{V}^{\prime}(V)=\frac{\bar{F}(V)+\sin ^{2} \alpha}{\bar{F}(V)} .
$$

Граничные условия для этой системы имеют вид

$$
R(0)=0 ; \quad F(0)=\cos ^{2} \alpha ; \quad \bar{V}(0)=0 ; \quad G(0)=0 .
$$

Как показано в работе [29], при выполнении неравенств

$$
\kappa_{1} \geq 3 \cdot 10^{-3} ; \quad \kappa_{2} \geq \kappa_{2 \min }\left(\kappa_{1}\right)=\frac{100}{1+0.8\left(\frac{\kappa_{1}}{0.2}\right)^{5}}
$$

для функции

$$
Q(V)=\sqrt{\frac{\kappa_{2}}{\pi}} \exp [-R(V)] \int_{0}^{V} \exp \left[R\left(V^{\prime}\right)\right] \frac{\Phi\left(V^{\prime}\right)}{\sqrt{V-V^{\prime}}} d V^{\prime}
$$

с точностью в несколько процентов справедливо приближение

$$
Q(V) \cong Q_{a}(V)=\Phi(V)^{0.5 b(V)} \operatorname{erf}[\sqrt{R(V)}] ;
$$

$$
\begin{gathered}
b(V)=1, \quad \text { если } V<a_{1} ; \\
b(V)=1-5\left[\left(\frac{V}{a_{1}}\right)^{\left.0.075\left(\frac{a_{1}}{V}\right)^{a_{2}}\left(\frac{500}{\kappa_{2}}\right)^{0.266}-1\right]}-1\right] \\
\times\left(\frac{V}{15}\right)^{a_{3}}\left(\frac{a_{4}}{\kappa_{1}}\right)^{0.7\left(\frac{\kappa_{1}}{0.1}\right)^{a_{5}}} ; \\
a_{1}=8.899 \cdot 10^{-4} \kappa_{2}+1.611 ; \\
a_{2}=-1.11 \cdot 10^{-4} \kappa_{2}+1.211 ; \\
a_{3}=2.788 \cdot 10^{-5} \kappa_{2}+0.072 ; \\
a_{4}=0.01 \quad \text { при } \quad \kappa_{1} \geq 0.005, \\
a_{4}=-2.5 \kappa_{1}+0.0225 \quad \text { при } \quad \kappa_{1}<0.005 ; \\
a_{5}=0.1 \quad \text { при } \quad \kappa_{1} \geq 0.005, \\
a_{5}=-40 \kappa_{1}+0.32 \quad \text { при } \quad \kappa_{1}<0.005 .
\end{gathered}
$$

Учитывая, что, как показывают расчеты, при $V>1.5$, $\kappa_{2} \gg 1$ при любых $\kappa_{1}, \alpha$ выполняется $F(V) \gg 1$ (т. е. $\left.\frac{[\bar{V}(v)-V]}{V} \ll 1\right)$, можно с той же степенью точности (и при тех же условиях) записать выражение для $\bar{N}_{2 i v}(V)$ в виде

$$
\begin{aligned}
& \bar{N}_{2 i v}(V) \cong \bar{N}_{2 i a}(V)=\left\{\frac { 1 } { \sqrt { F ( V ) } } \left[1+\kappa_{3}\left[\kappa_{2} G(V)\right.\right.\right. \\
& \left.\left.\left.+N_{e}(V)\right]\right]\right\}^{0.5 b(V)} \operatorname{erf}[\sqrt{R(V)}] .
\end{aligned}
$$

При использовании $\bar{N}_{2 i a}(V)$ вместо $\bar{N}_{2 i v}(V)$ во втором уравнении система (17) становится системой дифференциальных уравнений и решается стандартными численными методами в таких вычислительных программах, как Mathcad, Mathlab и т.п. Как показывают расчеты, при произвольных параметрах $\kappa_{1}$ и $\kappa_{2} \gg 1$ потенциалы $\bar{V}(V)$, $V$ для $V>10$ отличаются не более чем на $5 \%$.

Физические параметры: безразмерные ширины „предслоя“ $Y_{a}$ и ПС $Y_{w}-Y_{d} ; Z$-компонента относительной напряженности электрического поля $\frac{E(V)}{E}$ связаны с введенными функциями, входящими в систему (17), следующими формулами:

$$
Y_{d}=\kappa_{2} \int_{0}^{V_{d}} F(V)^{-0.5} d V
$$

$$
Y_{w}-Y_{d}=\kappa_{2} \int_{V_{d}}^{V_{w}} F(V)^{-0.5} d V ; \quad \frac{E(V)}{E}=F(V)^{0.5},
$$

где $V_{d}$ - безразмерный потенциал точки, которая определяется как граница между квазинейтрdальным „предслоем“ и ПС, где существенно нарушается квазинейтральность (находится как решение уравнения $\left.\frac{\partial n_{i}}{\partial V_{d}}=\frac{\partial n_{e}}{\partial V_{d}}\right) ; V_{w}-$ безразмерный потенциал стенки. 
Для средней скорости ионов, используя (11), получаем

$$
v_{i}(V)=v_{B} \frac{\left[1+\kappa_{3} \kappa_{2} G(V)\right]}{N_{i v}(V)} \sqrt{\frac{2}{\pi \kappa_{2}}} ; \quad v_{B}=\sqrt{\frac{k T_{e}}{M}} .
$$

При $\kappa_{3}=0$ это выражение совпадает с формулой, полученной в работе [29] в предположении отсутствия ионизации в ВПС.

Концентрация заряженных частиц в невозмущенной плазме $n_{0}$ связана с током $i_{f p}(T)$ на плоский зонд, находящийся под потенциалом $V \gg 1$, следующим соотношением:

$$
n_{0}=\sqrt{\frac{\pi \kappa_{2}}{2}} \frac{i_{f p}(V)}{e v_{B}\left[1+\kappa_{3} \kappa_{2} G(V)\right]\left[1+F_{S h}\left(\kappa_{1}, V\right)\right] S_{p}},
$$

где $F_{S h}\left(\kappa_{1}, V\right)$ - поправка на зависимость собирающей поверхности от потенциала плоского зонда, зависящая от величин $\kappa_{1}, V$ и полученная в работах [32,33], $S_{p}$ - площадь проводящей поверхности одностороннего зонда. Соотношение (23) следует рассматривать как уравнение для нахождения концентрации $n_{0}$ из экспериментально измеренной зависимости зондового тока от потенциала зонда. В правой части этого равенства функции $G(V), F_{S h}\left(\kappa_{1}, V\right)$ зависят от концентрации электронов, поскольку их значение определяется в том числе и параметром $\kappa_{1} \propto n_{0}^{-0.5}$. Параметры $\kappa_{3}, \kappa_{2}$ определяются из измеренных экспериментально значений $E, T_{e}$ и известного давления $P$. Уравнение решается методом последовательных приближений. В качестве нулевого приближения берется величина

$$
n_{00}=\sqrt{\frac{\pi \kappa_{2}}{2}} \frac{i_{f p}(V)}{e v_{B} S_{p}} .
$$

Приближения $n_{o j}$ и $n_{0 j-1}$ связаны соотношением

$$
\begin{aligned}
& n_{0 j}=\sqrt{\frac{\pi \kappa_{2}}{2}} \\
& \times \frac{i_{f p}(V)}{e v_{B}\left[1+\kappa_{3} \kappa_{2} G\left(V, \kappa_{1}\left(n_{0 j-1}\right)\right)\right]\left[1+F_{S h}\left(\kappa_{1}\left(n_{0 j-1}, V\right)\right)\right] S_{p}} .
\end{aligned}
$$

Решение модельных задач показало быструю сходимость метода, которая растет с уменьшением функций $G(V)$, $F_{S h}\left(\kappa_{1}, V\right)$.

Для учета зависимости сечения перезарядки $\sigma(\varepsilon)=\sigma_{0} B(\varepsilon)$ от энергии иона можно использовать метод последовательных приближений, который был применен в работе [28] при расчете ФРИ. В качестве нулевого приближения системы (17) достаточно в правой части первого уравнения (17) добавить множитель $B(V)$, в правой части второго уравнения (17) - вместо величины $R(V)$, определенной соотношением (15), использовать

$$
\bar{R}(V)=\int_{0}^{V} \frac{a\left(V^{\prime}\right) B\left(V^{\prime}\right)}{\sqrt{1-a\left(V^{\prime}\right)^{2} \sin ^{2} \alpha}} d V^{\prime},
$$

а под интегралом в правой части выражений для $\bar{N}_{2 i v}(V)$ и в формуле $(20)$ для $\bar{N}_{2 i a}(V)$ вместо $\frac{1}{\sqrt{F(V)}}-\frac{B(V)}{\sqrt{F(V)}}$. Процедура сходится очень быстро практически при любых параметрах $\kappa_{1}, \kappa_{2}$, так, что первое приближение практически не отличается от второго.

Для конкретных расчетов мы рассмотрим случай низкотемпературной газоразрядной плазмы инертных газов $\mathrm{He}$, Ar. Согласно [34], для частоты ионизации в этом случае справедливы аппроксимационные формулы зависимости от параметра $\frac{E}{P}$ соответственно

$$
\begin{aligned}
& v_{i \mathrm{He}}\left(\frac{E}{P}\right)=P(\text { Torr }) \cdot 5 \cdot 10^{6} \frac{E}{P}\left[1+5 \cdot 10^{-11}\left(\frac{E}{P}\right)^{3}\right] \\
& \times \exp \left[-14\left(\frac{E}{P}\right)^{-0.5}-1.7 \cdot 10^{-3} \frac{E}{P}\right] \\
& \text { при } 5 \frac{\mathrm{V}}{\mathrm{cm} \cdot \text { Torr }}<\frac{E}{P}<1000 \frac{\mathrm{V}}{\mathrm{cm} \cdot \text { Torr }} \text {; } \\
& v_{i \operatorname{Ar}}\left(\frac{E}{P}\right)=P(\text { Torr }) \cdot 2 \cdot 10^{7}\left(\frac{E}{P}\right)^{0.8} \\
& \times \exp \left[-28\left(\frac{E}{P}\right)^{-0.5}-1.9 \cdot 10^{-4} \frac{E}{P}\right] \\
& \text { при } 10 \frac{\mathrm{V}}{\mathrm{cm} \cdot \text { Torr }}<\frac{E}{P}<10000 \frac{\mathrm{V}}{\mathrm{cm} \cdot \text { Torr }} \text {. }
\end{aligned}
$$

В этих формулах частота ионизации выражена в $\mathrm{s}^{-1}$, параметр $\frac{E}{P}$ в $\frac{\mathrm{V}}{\mathrm{cm} \cdot \text { Torr }}$ Экспериментальные результаты авторов $[35,36]$ по дрейфовой скорости ионов в собственном газе для Не и [35-37] для Ar хорошо аппроксимируются соотношениями

$$
\begin{aligned}
v_{i d \mathrm{He}}\left(\frac{E}{P}\right) & =0.915 \cdot\left(\frac{E}{P}\right)^{\alpha_{\mathrm{He}}\left(\frac{E}{P}\right)} ; \\
\alpha_{\mathrm{He}}\left(\frac{E}{P}\right) & =\frac{0.95}{\left(1+\frac{E}{27 P}\right)^{0.09}} \\
v_{i d \mathrm{Ar}}\left(\frac{E}{P}\right) & =0.1 \cdot\left(\frac{E}{P}\right)^{\alpha_{\mathrm{Ar}}\left(\frac{E}{P}\right)} \\
\alpha_{\mathrm{Ar}}\left(\frac{E}{P}\right) & =\frac{1.05}{\left(1+\frac{E}{40 P}\right)^{0.081}}
\end{aligned}
$$

соответственно, где дрейфовая скорость выражена в $\frac{\mathrm{cm}}{\mathrm{s}}$, а $\frac{E}{P}$ в $\frac{\mathrm{V}}{\mathrm{cm} \cdot \text { Torr }}$. С учетом соотношений (5), (7) для параметра $\kappa_{3}$ в случае Не, Ar получаем

$$
\kappa_{3 \mathrm{He}}=1.1 \cdot 10^{-6} \frac{v_{i \mathrm{He}}\left(\frac{E}{P}\right)}{v_{i d \mathrm{He}}\left(\frac{E}{P}\right)} ; \quad \kappa_{3 \mathrm{Ar}}=5.6 \cdot 10^{-7} \frac{v_{i \mathrm{Ar}}\left(\frac{E}{P}\right)}{v_{i d \mathrm{Ar}}\left(\frac{E}{P}\right)}
$$

соответственно. Параметр $\frac{E}{P}$ в $(26)$ относится к невозмущенной плазме. При получении соотношений (27) 

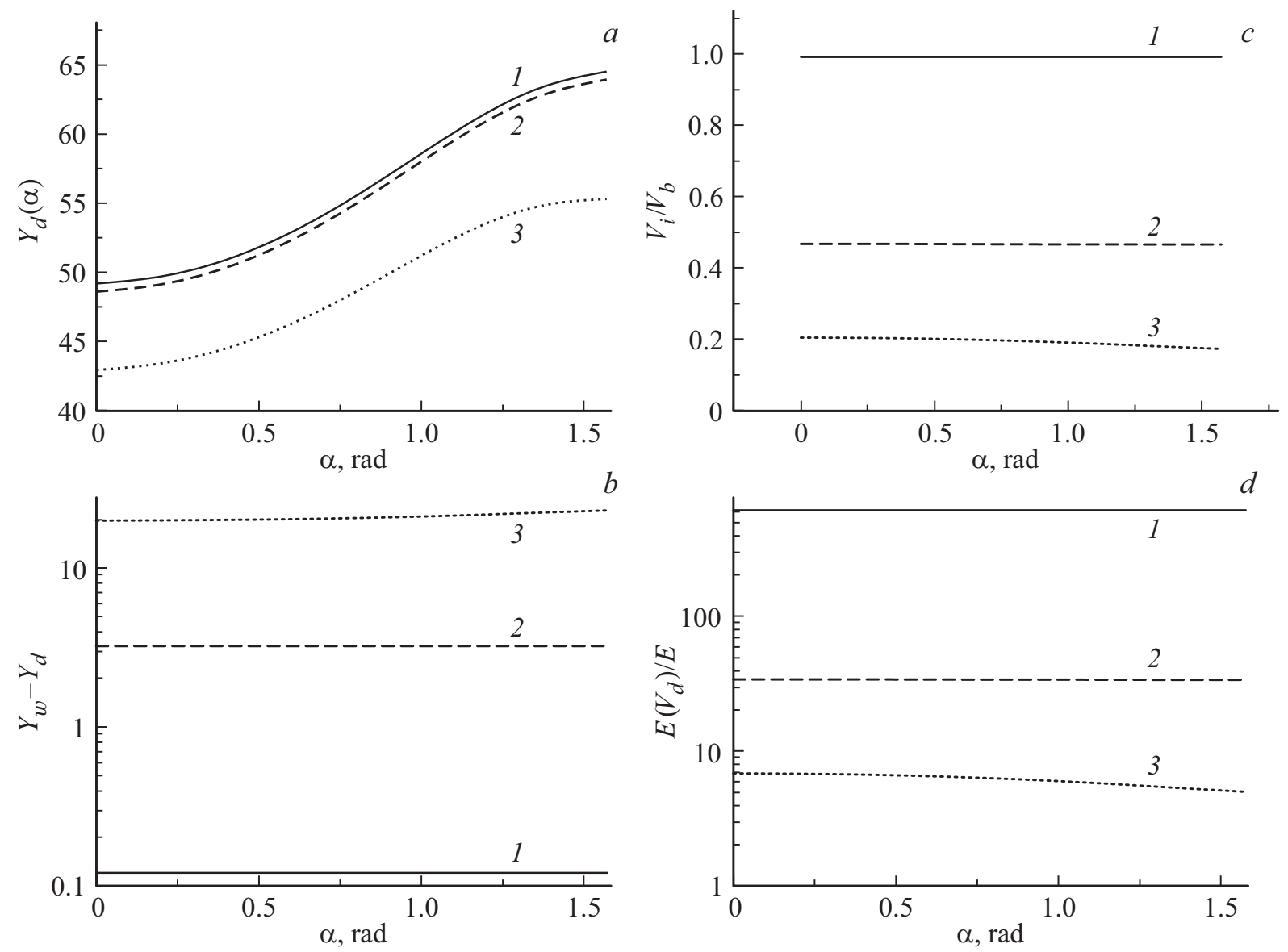

Рис. 2. Зависимость протяженности квазинейтрального „предслоя“ $Y_{d}(a)$; протяженности пристеночного слоя $Y_{w}-Y_{d}(b)$; относительной скорости ионов $v_{i} / v_{b}$ на границе „предслоя“ и ПС $(c)$; относительной $Z$-составляющей электрического поля $E\left(V_{d} / E\right)$ на границе „предслоя“ и ПС от угла $\alpha$ между нормалью к возмущающей поверхности и вектором электрического поля в невозмущенной плазме при различных параметрах $\kappa_{1}$ и $\kappa_{2}=100(d) ; 1-\kappa_{1}=0.003 ; 2-0.1 ; 3-1$.

использовались данные о сечениях резонансной перезарядки ионов $\mathrm{He}^{+}, \mathrm{Ar}^{+}$, приведенные в [38].

Для учета зависимости сечений резонансной перезарядки ионов $\mathrm{He}^{+}, \mathrm{Ar}^{+}$, от энергии относительного движения иона и атома, согласно [38], достаточно положить $\sigma_{0 \mathrm{He}}=2.79 \cdot 10^{-15} \mathrm{~cm}^{2} ; \sigma_{0 \mathrm{Ar}}=5.53 \cdot 10^{-15} \mathrm{~cm}^{2}$; $B\left(\varepsilon_{i}\right)=\left[1+a \ln \left(\varepsilon_{i}\right)\right]^{2} ; \quad a_{\mathrm{He}}=0.0577 ; \quad a_{\mathrm{Ar}}=0.0543$, где $\varepsilon_{i}$ - относительная энергия иона и атома, выраженная в $\mathrm{eV}$. Учитывая, что в рассматриваемых условиях скорость иона много больше скорости атома, можно считать, что $\varepsilon_{i}$ близка к энергии иона.

\section{Обсуждение полученных результатов}

В работах $[28,29]$ было получено решение задачи о структуре ВПС в ситуации, когда угол $\alpha=0$ и отсутствует ионизация. Рассмотрим сначала зависимость параметров ВПС от угла $\alpha$, пренебрегая ионизацией. Поскольку, как показано в [29], структура ВПС слабо зависит от вида ФРЭ и определяется в основном их средней энергией, то при расчетах будем считать, что
ФРЭ - максвелловская, т. е.

$$
N_{e}(V)=\exp (-V) \text {. }
$$

Как упоминалось ранее, ВПС состоит из двух слоев квазинейтрального „Предслоя“ и ПС, в котором существенно нарушается квазинейтральность. Параметры ионного потока на границе этих двух слоев существенно зависят от параметра $\kappa_{1}$ и не слишком сильно от отношения средних энергий электронов и ионов $\kappa_{2}$ (при условии, что $\left.\kappa_{2} \gg 1\right)$. В широком диапазоне изменения указанных параметров средняя энергия ионов на границе „Предслоя“ и ПС оказывается много больше таковой в невозмущенной плазме. Увеличение скорости ионов в „предслое“ происходит за счет возрастания электрического поля в направлении нормали к возмущающей поверхности. Вполне естественно, что если поле в невозмущенной плазме направлено параллельно поверхности, то формирование достаточно большого электрического поля в ортогональном направлении потребует большей протяженности „предслоя“, чем в случае, когда поле в невозмущенной плазме направлено по нормали к поверхности. Таким образом, можно ожидать, что струк- 


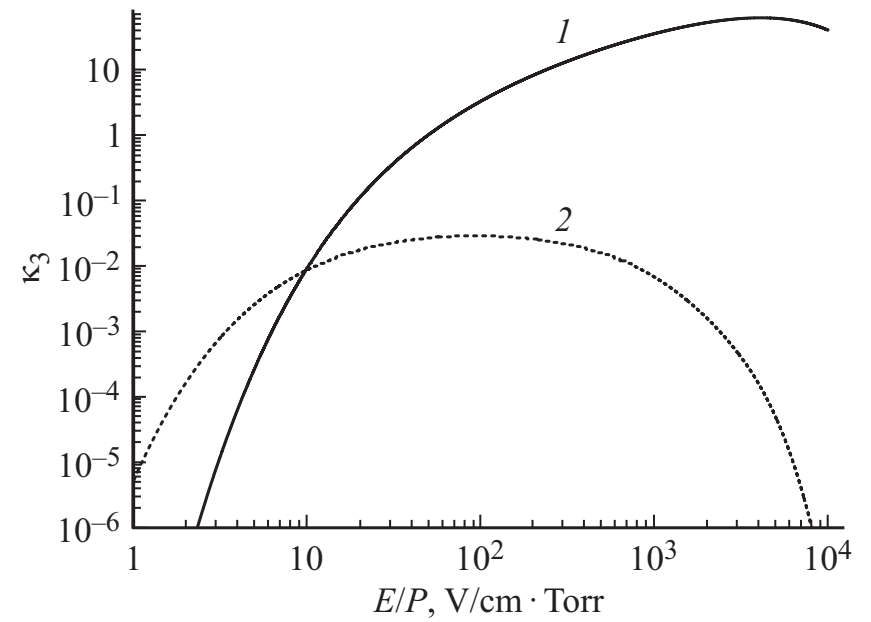

Рис. 3. Зависимость параметра $\kappa_{3}$ от величины $E / P ; 1$ - для плазмы $\mathrm{Ar} ; 2$ - для плазмы Не.

тура „предслоя“ будет зависеть от угла $\alpha$. В то же время при значительных потенциалах $V$ электрическое поле в ПС существенно превосходит поле в ,предслое“ и тем более в невозмущенной плазме. Поэтому структура ПС не должна зависеть от ориентации поля в невозмущенной плазме (при выполнении условия $\kappa_{2} \gg 1$ ).

Расчеты параметров ВПС на рис. 2 сделаны нами для случая зависимости сечения перезарядки ионов $\mathrm{Ar}^{+}$от энергии иона.

На рис. 2, $a$ приведена зависимость от угла протяженности „предслоя“ $Y_{d}$ при различных параметрах $\kappa_{1}$ и $\kappa_{2}=100$. Как и предполагалось, с ростом угла протяженность „предслоя“ возрастает.

Аналогичные зависимости, но для протяженности ПС $Y_{w}-Y_{d}$, приведены на рис. $2, b$. Видно, что при малых $\kappa_{1} \ll 1$, величина $Y_{d}$ практически не зависит от угла $\alpha$. При $\kappa_{1} \cong 1$ появляется слабая угловая зависимость, которая растет с увеличением параметра $\kappa_{1}$. Выше было приведено физическое обоснование такого поведения величины $Y_{w}-Y_{d}$.

Данные рис. 2, $c, d$ показывают слабую зависимость средней скорости ионов $v_{i}\left(V_{d} / v_{B}\right)$ и величины $Z$-компоненты электрического поля $E\left(V_{d}\right) / E$ на границе ,предслоя“" и ПС от угла $\alpha$. Видно, что с ростом параметра $\kappa_{1}$ зависимость становится более заметной. А именно скорость и электрическое поле с ростом угла $\alpha$ уменьшаются.

Теперь рассмотрим влияние ионизации в ВПС на его структуру. На рис. 3 приведена зависимость величин $\kappa_{3 \mathrm{He}}, \kappa_{3 \mathrm{Ar}}$ от параметра $\frac{E}{P}$. Видно, что с ростом этого параметра рассчитываемые величины возрастают и, пройдя максимум, уменьшаются. Отметим, что при $\frac{E}{P}<10$ выполняется $\kappa_{3 \mathrm{He}}, \kappa_{3 \mathrm{Ar}}<0.01$. При этом, согласно данным [5], в неравновесной плазме в Не при $P L>0.5$ выполняется $\frac{E}{P}<5$, а в $\operatorname{Ar} \frac{E}{P}<2$, где $L-$ характерный размер плазмы. Аналогичные неравенства справедливы и для других инертных газов. Таким обра- зом, можно констатировать, что в достаточно широком диапазоне условий в неравновесной газоразрядной плазме инертных газов выполняется соотношение $\kappa_{3} \ll 1$.

На рис. 4-10 приведены сравнительные расчеты параметров ВПС с учетом ионизации и без нее для газоразрядной плазмы He, Ar. Видно, что существует диапазон параметров плазмы, где учет ионизации существенно
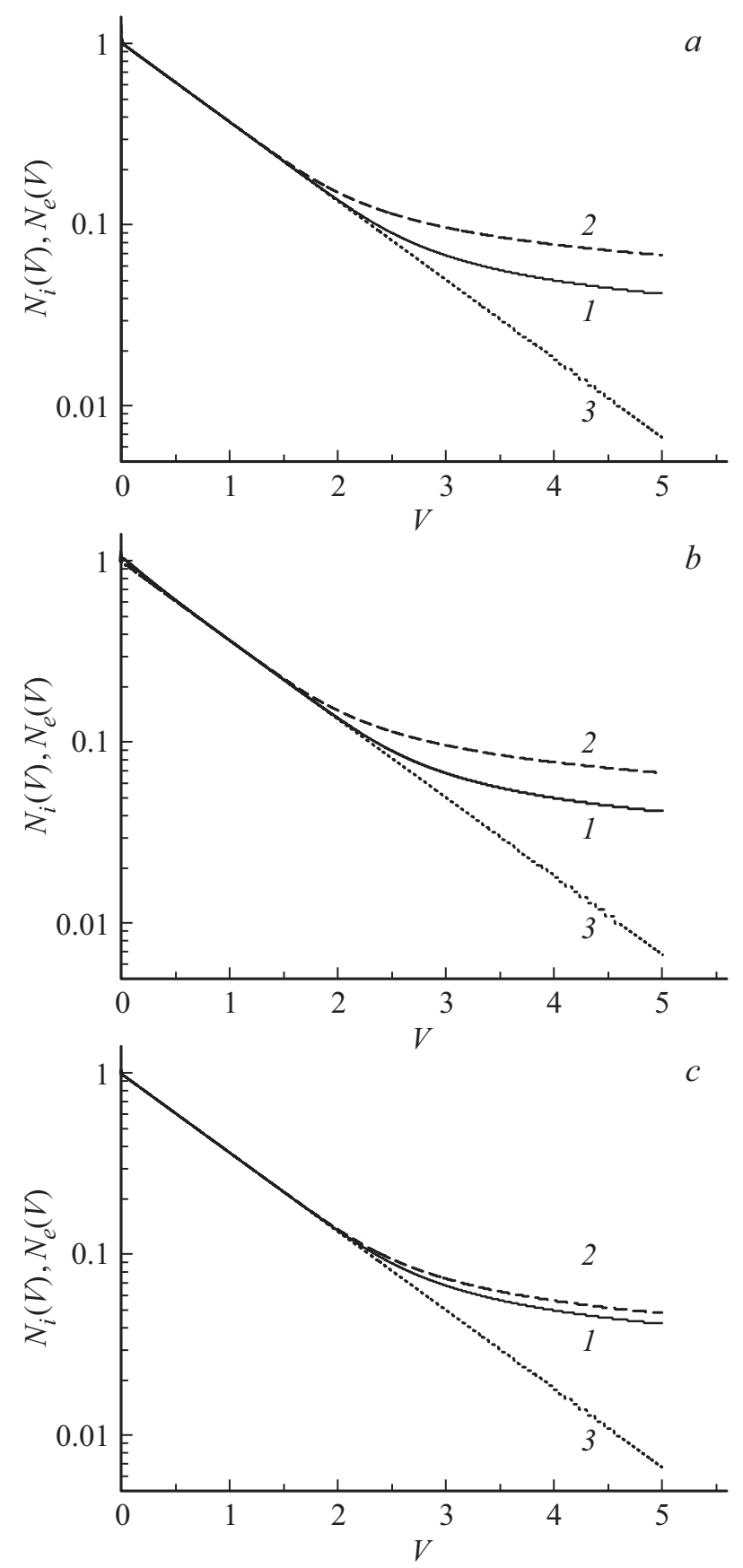

Рис. 4. Зависимость концентрации ионов в ВПС для неравновесной плазмы $\operatorname{Ar}(a-\alpha=0 ; b-\alpha=\pi / 4)$ и Не $(c-\alpha=0)$ от безразмерного потенциала пространства при следующих условиях: $\frac{E}{P}=30 \frac{\mathrm{v}}{\mathrm{cm} \cdot \text { Torr }} ; \kappa_{1}=0.05 ; \kappa_{2}=100 ; V_{w}=15 ; 1-$ $N_{i}(V)$ без учета ионизации в ВПС; $2-N_{i}(V)$ с учетом ионизации в ВПС; $3-N_{e}(V)$. 


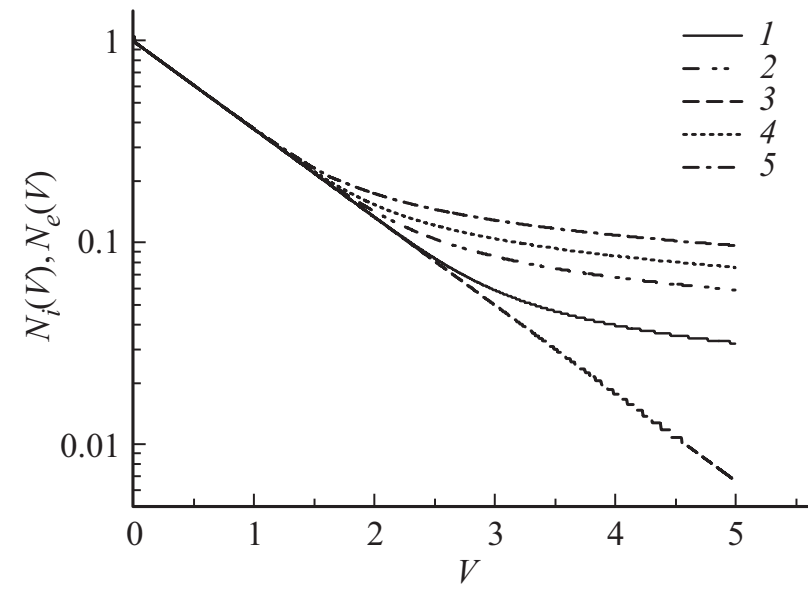

Pис. 5. Зависимость концентрации ионов в ВПС неравновесной плазмы $\mathrm{Ar}$ от безразмерного потенциала пространства при следующих условиях: $\frac{E}{P}=30 \frac{\mathrm{v}}{\mathrm{cm} \cdot \mathrm{Torr}} ; \kappa_{1}=0.05 ; \alpha=0 ; V_{w}=15$; $\kappa_{2}=200: 1-N_{i}(V)$ без учета ионизации в ВПС; $2-$ с учетом ионизации в ВПС; $3-N_{e}(V) ; \kappa_{2}=20 ; 4-$ без учета ионизации в ВПС; $5-N_{i}(V)$ с учетом ионизации в ВПС.

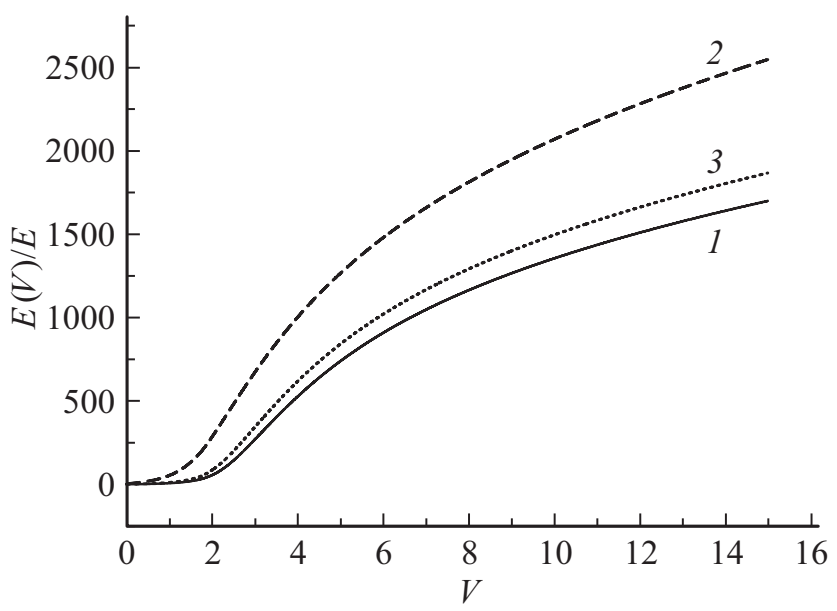

Рис. 6. Зависимость относительного электрического поля в ВПС от безразмерного потенциала; $\kappa_{1}=0.05 ; \alpha=0 ; V_{w}=15$; $\kappa_{2}=100 ; 1-$ без учета ионизации в ВПС; 2 - с учетом ионизации в ВПС для плазмы $\mathrm{Ar}$ при $\frac{E}{P}=30 \frac{\mathrm{V}}{\mathrm{cm} \cdot \text { Torr }} ; 3-$ с учетом ионизации в ВПС для плазмы Не при $\frac{E}{P} \stackrel{\mathrm{cm} \cdot \text { Torr }}{=} 30 \frac{\mathrm{V}}{\mathrm{cm} \cdot \text { Torr }}$.

изменяет структуру ВПС. Обсудим физические причины этого.

На рис. 4, $a, c$ сравнивается зависимость относительной концентрации ионов при $\kappa_{1}=0.05 ; \kappa_{2}=100 ; \alpha=0$; $V_{w}=15, \frac{E}{P}=30 \frac{\mathrm{V}}{\mathrm{cm} \cdot \mathrm{Torr}}$, от безразмерного потенциала в ВПС для $\mathrm{Ar}, \mathrm{He}$ соответственно. Как и следовало ожидать, в обоих случаях концентрация при учете ионизации выше. При этом отличие для Ar существенно больше. Последнее обусловлено тем, что, согласно данным рис. 6, при этом значении приведенного поля $\frac{E}{P}$ параметр $\kappa_{3}$ для Ar заметно выше, чем для Не.

Аналогичные данные, но для $\operatorname{Ar}$ и угла $\alpha=\pi / 4$ приведены на рис. $4, b$. Видно, что наблюдается слабая зависимость результатов расчетов от угла между полем в плазме и нормалью к поверхности. Это можно объяснить аналогично тому, как было сделано для случая отсутствия ионизации.

Из данных, приведенных на рис. 5, где рассчитана зависимость концентрации заряженных частиц от безразмерного потенциала при различных параметрах $\kappa_{2}$ с учетом ионизации и без нее, следует, что влияние ионизации растет с увеличением этого параметра. Это связано с тем, что рост параметра $\kappa_{2}$ означает уменьшение средней энергии ионов в невозмущенной плазме, в силу чего (как было показано ранее) протяженность квазинейтрального „предслоя“ возрастает, а значит, возрастает и количество актов ионизации в этой части ВПС.

Следствием роста концентрации ионов при сохранении концентрации электронов является более существенное нарушение квазинейтральности (как в ,предслое“, так и в ПС) и, следовательно, рост электрическо-

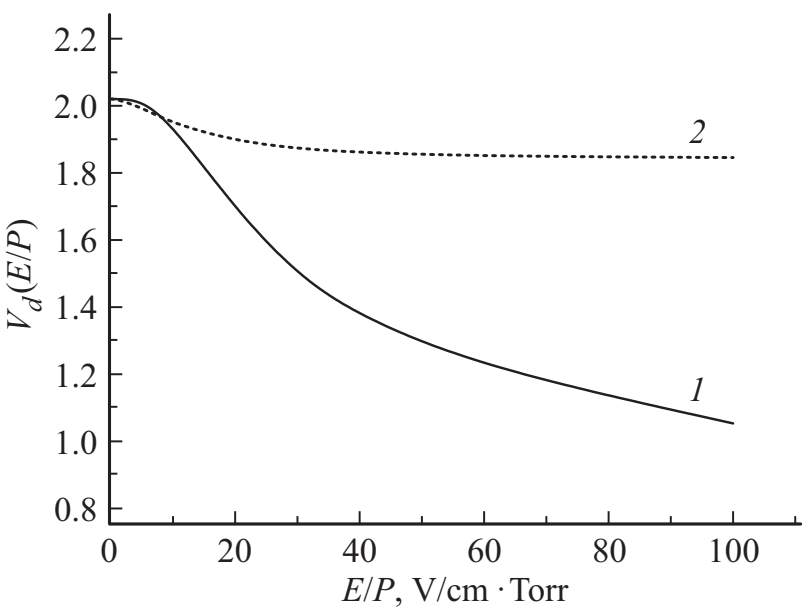

Рис. 7. Зависимость потенциала на границе „предслоя“ и ПС от параметра $E / P$ при $\kappa_{1}=0.05 ; \alpha=0 ; V_{w}=15 ; \kappa_{2}=100$; 1 - для плазмы $\mathrm{Ar} ; 2$ - для плазмы Не.

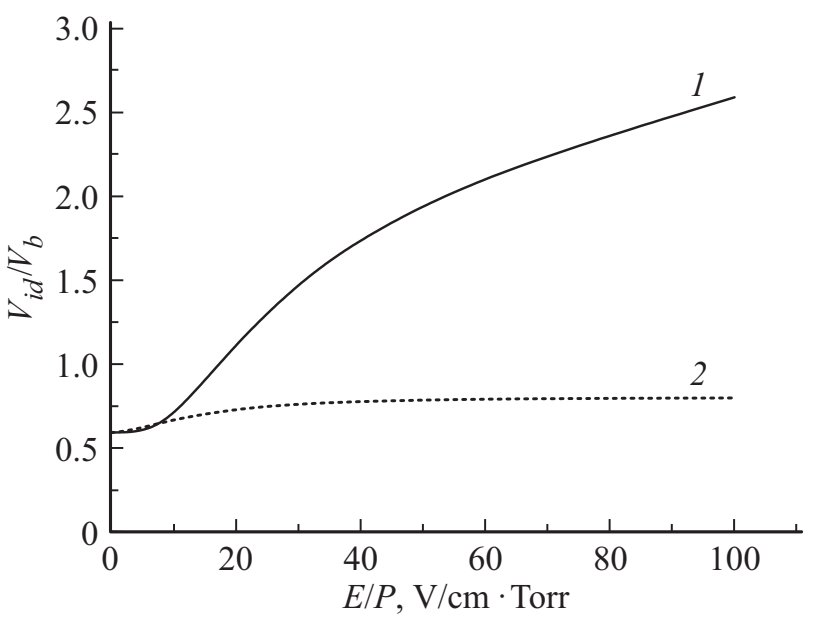

Рис. 8. Зависимость средней относительной скорости ионов на границе „предслоя“ и ПС от параметра $E / P$ при $\kappa_{1}=0.05$; $\alpha=0 ; V_{w}=15 ; \kappa_{2}=100 ; 1-$ для плазмы $\mathrm{Ar} ; 2-$ для плазмы Не. 


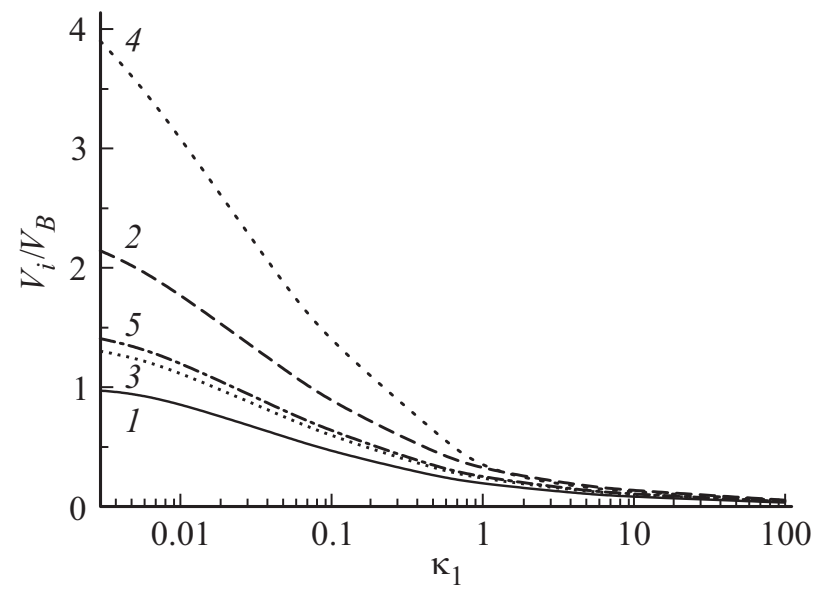

Рис. 9. Зависимость средней относительной скорости ионов на границе „предслоя“ и ПС от параметра $\kappa_{1}$ при различных $E / P$ для $\mathrm{He}$ и $\mathrm{Ar}$ и $\alpha=0 ; \kappa_{2}=100: 1-\frac{E}{P}=0, \mathrm{He}, \mathrm{Ar}$; $2-\frac{E}{P}=20 \frac{\mathrm{V}}{\mathrm{cm} \cdot \operatorname{Torr}}, \quad$ Ar; $3-\frac{E}{P}=20 \frac{\mathrm{V}}{\mathrm{cm} \cdot \text { Torr }}, \mathrm{He} ; 4-$ $\frac{E}{P}=40 \frac{\mathrm{V}}{\mathrm{cm} \cdot \operatorname{Torr}}, \operatorname{Ar} ; 5-\frac{E}{P}=40 \frac{\mathrm{V}}{\mathrm{cm} \cdot \text { Torr }}, \mathrm{He}$.

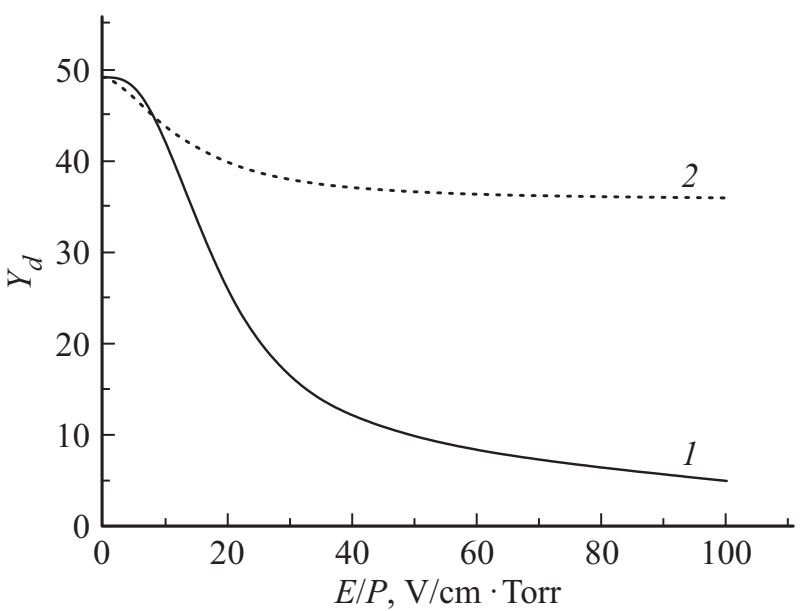

Рис. 10. Зависимость протяженности „предслоя“ от параметра $E / P$ при $\kappa_{1}=0.05 ; \alpha=0 ; V_{w}=15 ; \kappa_{2}=100 ; 1-$ для плазмы $\mathrm{Ar} ; 2$ - для плазмы Не.

го поля в ВПС. Это иллюстрируется данными рис. 6, где приведена зависимость относительного электрического поля от безразмерного потенциала в ВПС для плазмы $\mathrm{He}, \mathrm{Ar}$ при $\kappa_{1}=0.05 ; \alpha=0 ; V_{w}=15 ; \kappa_{2}=100$, $\frac{E}{P}=30 \frac{\mathrm{V}}{\mathrm{cm} \cdot \text { Torr }}$.

Зависимость безразмерного потенциала на границе „предслоя“ и ПС (определенной, как точка равенства производных ионов и электронов по потенциалу) от параметра $E / P$ в невозмущенной плазме при $\kappa_{1}=0.05$; $\alpha=0 ; V_{w}=15 ; \kappa_{2}=100$ для плазмы $\mathrm{He}, \mathrm{Ar}$ представлена на рис. 7. Видно, что с ростом $E / P$, т.е. при увеличении роли ионизации в ВПС, потенциал $V_{d}$ падает. Это происходит в силу того, что из-за наличия ионизации в „предслое“ нарушение квазинейтральности происходит раньше, что приводит к резкому уменьше- нию протяженности „предслоя“ (рис. 9) и, несмотря на рост поля - к падению $V_{d}$.

На рис. 8 приведена зависимость средней скорости ионов (в единицах бомовской скорости $v_{B}$ ) от параметра $E / P$ в невозмущенной плазме при $\kappa_{1}=0.05 ; \alpha=0$; $V_{w}=15 ; \kappa_{2}=100$ для плазмы $\mathrm{He}, \mathrm{Ar}$, а на рис. $9-$ зависимость этой величины от параметра $\kappa_{1}$ при различных $E / P$ для плазмы Не, Ar. Видно, что с ростом параметра $E / P$ относительная скорость ионов $v_{i d / v_{B}}$ увеличивается. При этом если при $\frac{E}{P}=0$ и $\kappa_{1} \rightarrow 0$ выполняется $v_{i d} \rightarrow v_{d}$, то при учете ионизации средняя скорость ионов $v_{i d}$ при $\kappa_{1} \rightarrow 0$ сушественно превосходит бомовскую. Причина этого заключается в том, что увеличение $E / P$ в невозмущенной плазме, приводящее к росту частоты ионизации, вызывает резкое повышение электрического поля в ВПС и, таким образом, рост

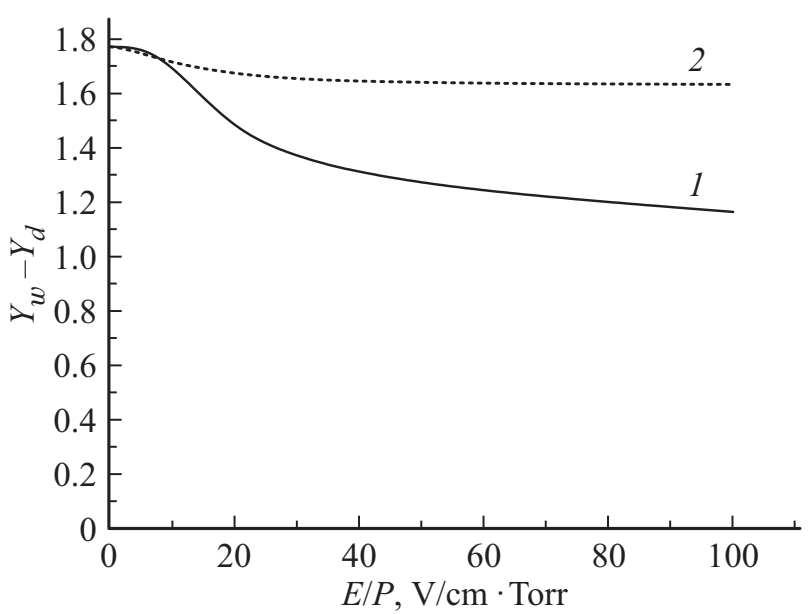

Рис. 11. Зависимость протяженности ПС от параметра $E / P$ при $\kappa_{1}=0.05 ; \alpha=0 ; V_{w}=15 ; \kappa_{2}=100 ; 1$ - для плазмы $\mathrm{Ar}$; 2 - для плазмы Не.

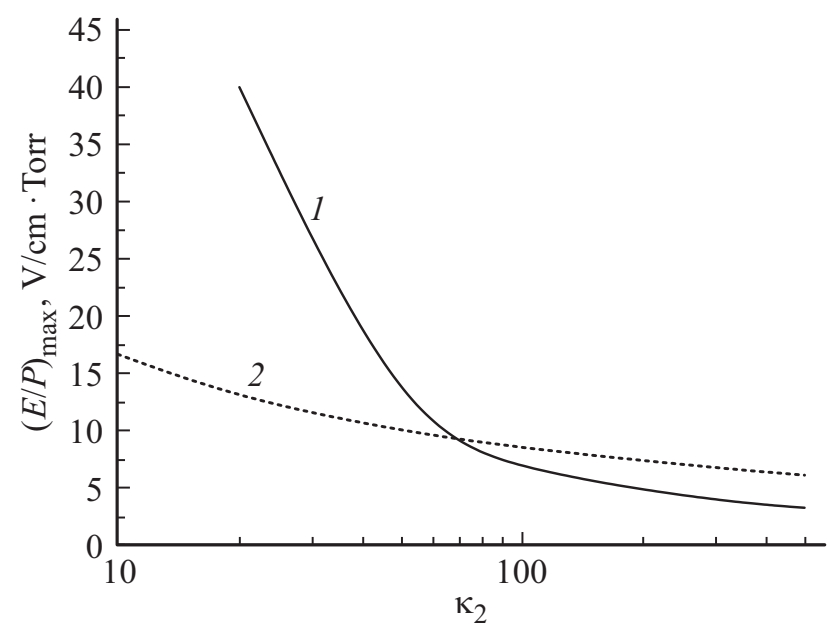

Рис. 12. Зависимость от параметра $\kappa_{2}$ максимального значения приведенного поля $(E / P)_{\max }$ в невозмущенной плазме, при котором (независимо от значения параметра $\kappa_{1}$ ) еще можно не учитывать ионизацию при расчете структуры ВПС; 1 - для плазмы $\mathrm{He} ; 2$ - для плазмы $\mathrm{Ar}$. 
средней скорости ионов. Кроме того, как показывают расчеты, величина $v_{i d} / v_{B}$ при значениях $E / P$, при которых требуется учет ионизации, возрастает с увеличением параметра $\kappa_{2}$ существенно сильнее, чем при $\frac{E}{P}=0$. Это обусловлено тем, что, как было показано, при увеличении $\kappa_{2}$ растет протяженность „предслоя“, что увеличивает влияние ионизации, вызывающей рост $v_{i d} / v_{B}$

Как видно из данных рис. 10, 11, где представлены зависимости протяженности „предслоя“ и ПС соответственно от параметра $E / P$ для плазмы $\mathrm{He}, \mathrm{Ar}$ при $\kappa_{1}=0.05 ; \alpha=0 ; V_{w}=15 ; \kappa_{2}=100$, рост ионизации в ВПС приводит к резкому уменьшению протяженности „предслоя“ и менее существенному уменьшению протяженности ПС. Это, видимо, обусловлено тем, что ПС имеет протяженность порядка радиуса Дебая, поэтому число актов ионизации в этой части ВПС остается небольшим. В то же время протяженность „предслоя“ может быть велика, поэтому рост частоты ионизации здесь существенно увеличивает концентрацию ионов, что и приводит к наблюдаемой более резкой зависимости протяженности „предслоя“ от параметра $E / P$.

Расчеты показывают, что параметр $\kappa_{1}$ слабо влияет на изменения параметров ВПС при учете ионизации, в то время как влияние величины $\kappa_{2}$ на эти параметры существенно. На рис. 12 приведены зависимости максимального приведенного электрического поля в невозмущенной плазме $(E / P)_{\max }$, при котором еще можно не учитывать ионизацию в ВПС, от параметра $\kappa_{2}$ для плазмы He, Ar. Видно, что при уменьшении параметра $\kappa_{2}$ величина $(E / P)_{\max }$ возрастает. Причины этого мы объяснили выше. При этом для случая Не при $\kappa_{2}<20$ величина $(E / P)_{\max }$ неограниченно возрастает. Это связано с наличием максимума на зависимости параметра $\kappa_{3}(E / P)$ вблизи значения $\frac{E}{P} \approx 40 \frac{\mathrm{V}}{\mathrm{cm} \cdot \text { Torr }}$, после которого $\kappa_{3}$ начинает уменьшаться.

\section{Заключение}

Как мы видели, при условии, что $\kappa_{2} \gg 1$, которое, как правило, реализуется в неравновесной газоразрядной плазме, структура ВПС около плоской поверхности, находящейся под отрицательным потенциалом, существенно превосходящим среднюю энергию электронов, слабо зависит от взаимной ориентации электрического поля в невозмущенной плазме и нормали к поверхности.

Кроме того, показано, что ионизация в ВПС может заметно влиять на структуру как квазинейтрального „предслоя“, так и ПС, где существенно нарушается квазинейтральность. Особенно это относится к „предслою“, протяженность которого намного больше, чем ПС. Так, средняя скорость ионов на границе „предслоя“ и ПС при наличии ионизации в ,предслое“ может существенно превосходить бомовскую при режимах, близких к пролетным (при малых параметрах $\kappa_{1}$ ). Ранее считалось, что максимум этой скорости не превосходит скорости Бома.
Обычно при анализе роли ионизации в формировании структуры пристеночного слоя авторы, как правило, отмечали, что протяженность ПС обычно намного меньше, чем длина пробега электрона относительно процесса ионизации, а об ионизации в „предслое“ ничего не говорилось.

Таким образом, в газоразрядной плазме существует диапазон параметров, где учет этого явления необходим. В частности, в плазме инертных газов при параметре $\frac{E}{P}$ порядка десяти $\frac{\mathrm{V}}{\mathrm{cm} \cdot \mathrm{Torr}}$ и более при зондовых измерениях концентрации заряженных частиц по ионному току насыщения необходимо учитывать увеличение плотности тока и изменение структуры ВПС из-за роста концентрации ионов и дрейфовой скорости в результате ионизации в ,предслое“.

\section{Конфликт интересов}

Авторы заявляют, что у них нет конфликта интересов.

\section{Список литературы}

[1] Donnelly V.M., Kornblit A. // J. Vacuum Sci. Technol. A: Vacuum, Surfaces, Films. 2013. Vol. 31. N 5. P. 050825.

[2] Sturges D.J., Oskam H.J. // J. Appl. Phys. 1964. Vol. 35. N 10. P. 2887-2894.

[3] Rubel M. et al. // J. Nucl. Mater. 1992. Vol. 196. P. 285-291.

[4] Дунаев В.В., Жиглинский А.Г., Сухомлинов В.С., Фабурина Э.Н. // ЖТФ. 1992. Т. 62. Вып. 2. С. 41-46.

[5] Raizer Y. Gas Discharge Physics. Berlin, NY.: Springer, 1991. P. 536.

[6] Chen X.P. // Phys. Plasmas. 1998. Vol. 5. N 3. P. 804-807.

[7] Bohm D. The use of probes for plasma exploration in strong magnetic fields // Characteristics of Electrical Discharges in Magnetic Fields. 1949. Vol. 13.

[8] Riemann K. // Plasma Sources Sci. Technol. 2009. Vol. 18. P. 014006.

[9] Benilov M. // Plasma Sources Sci. Technol. 2008. Vol. 18. N 1. P. 014005.

[10] Allen J. // Plasma Sources Sci. Technol. 2009. Vol. 18.P. 014004.

[11] Brinkmann R. // J. Phys. D: Appl. Phys. 2011. Vol. 44. N 4. P. 042002.

[12] Kino G., Shaw E. // Phys. Fluid. 1966. Vol. 9. N 3. P. 587-593.

[13] Chen F.F. Introduction to plasma physics. NY., London: Plenum Press, 1974.

[14] Tsankov T., Czarnetzki U. // Plasma Sour. Sci. Technol. 2017. Vol. 26. N 5. P. 055003.

[15] Kos L., Tskhakaya S., Jelic N. // Phys. Plasmas. 2015. Vol. 22. N 9. P. 093503.

[16] Riemann K. // J. Phys. D: Appl. Phys. 2003. Vol. 36. N 22. P. 2811.

[17] Riemann K. // J. Phys. D: Appl. Phys. 1991. Vol. 24. N 4. P. 493.

[18] Baalrud S., Hegna C. // Plasma Sour. Sci. Technol. 2011. Vol. 20. N 2. P. 025013.

[19] Riemann K. // Phys. Plasmas. 2006. Vol. 13. N 6. P. 063508.

[20] Godyak V. // Phys. Lett. A. 1982. Vol. 89. N 2. P. 80-81. 
[21] Valentini H. // Phys. Plasmas. 1996. Vol. 3. N 4. P. $1459-1461$.

[22] Chen X. // Phys. Plasmas. 1998. Vol. 5. N 3. P. 804-807.

[23] Kaganovich I. // Phys. Plasmas. 2002. Vol. 9. N 11. P. $4788-4793$.

[24] Riemann K. // Phys. Fluids. 1981. Vol. 24. N 12. P. $2163-2172$.

[25] Raoul N. // IEEE Transactions on Plasma Science. 2002. Vol. 30. N 1. P. 352-356.

[26] Godyak V., Sternberg N. // IEEE Transactions on Plasma Science. 2003. Vol. 31. N 2. P. 303.

[27] Franklin R.N. // J. Phys. D: Appl. Phys. 2003. Vol. 36. N 22. P. 2821.

[28] Sukhomlinov V.S., Mustafaev A.S., Murillo O. // Phys. Plasmas. 2018. Vol. 25. N 1. P. 013513.

[29] Мурильо О., Мустабаев А.С., Сухомлинов В.С. // ЖТФ. 2019. Т. 89. Вып. 9.

[30] Мустафаев А.С., Сухомлинов В.С., Аинов М. // ЖТФ. 2015. T. 60. C. 1778.

[31] Mustafaev A., Sukhomlinov V., Ainov M. // High Temperature. 2017. Vol. 55. N 3. P. 346- 351.

[32] Sheridan T. // Phys. Plasmas. 2000. Vol. 7. N 7. P. 3084-3088.

[33] Sheridan T. // J. Phys. D: Appl. Phys. 2010. Vol. 43. N 10. P. 105204.

[34] Ткачев А., Яковленко С. // Труды ИОФАН. 2007. Т. 63. C. 64-101.

[35] Hornbeck A. // Phys. Rev. 1951. Vol. 84. N 4. P. 615.

[36] МакДаниэль E., Масон E. // Нью Йорк-Лондон-Сидней-Торонто: Джон Вилей и сыновья, 1973. C. 320 .

[37] Madson J., Oskam H. // Phys. Lett. A. 1967. Vol. 25. N 5. P. 407-408.

[38] Maiorov S., Petrov O., Fortov V. // Proc. of the 34th EPS Conf. on Plasma Physics, Warsaw. 2007.

URL: http://epsppd.epfl.ch/Warsaw/pdf2/P2_115.pdf 